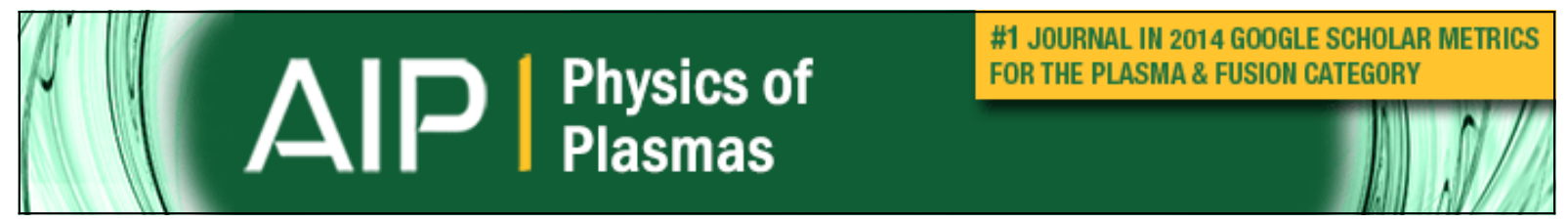

The ion velocity distribution function in a current-free double layer

Amy M. Keesee, Earl E. Scime, Christine Charles, Albert Meige, and Rod Boswell

Citation: Physics of Plasmas 12, 093502 (2005); doi: 10.1063/1.2033647

View online: http://dx.doi.org/10.1063/1.2033647

View Table of Contents: http://scitation.aip.org/content/aip/journal/pop/12/9?ver=pdfcov

Published by the AIP Publishing

Articles you may be interested in

Ion acceleration in $\mathrm{Ar}-\mathrm{Xe}$ and $\mathrm{Ar}-\mathrm{He}$ plasmas. II. Ion velocity distribution functions

Phys. Plasmas 17, 113509 (2010); 10.1063/1.3505823

Laser-induced-fluorescence observation of ion velocity distribution functions in a plasma sheath

Phys. Plasmas 13, 062103 (2006); 10.1063/1.2206786

High source potential upstream of a current-free electric double layer

Phys. Plasmas 12, 044508 (2005); 10.1063/1.1883182

Time development of a current-free double-layer

Phys. Plasmas 11, 3808 (2004); 10.1063/1.1764829

Current-free double-layer formation in a high-density helicon discharge

Appl. Phys. Lett. 82, 1356 (2003); 10.1063/1.1557319

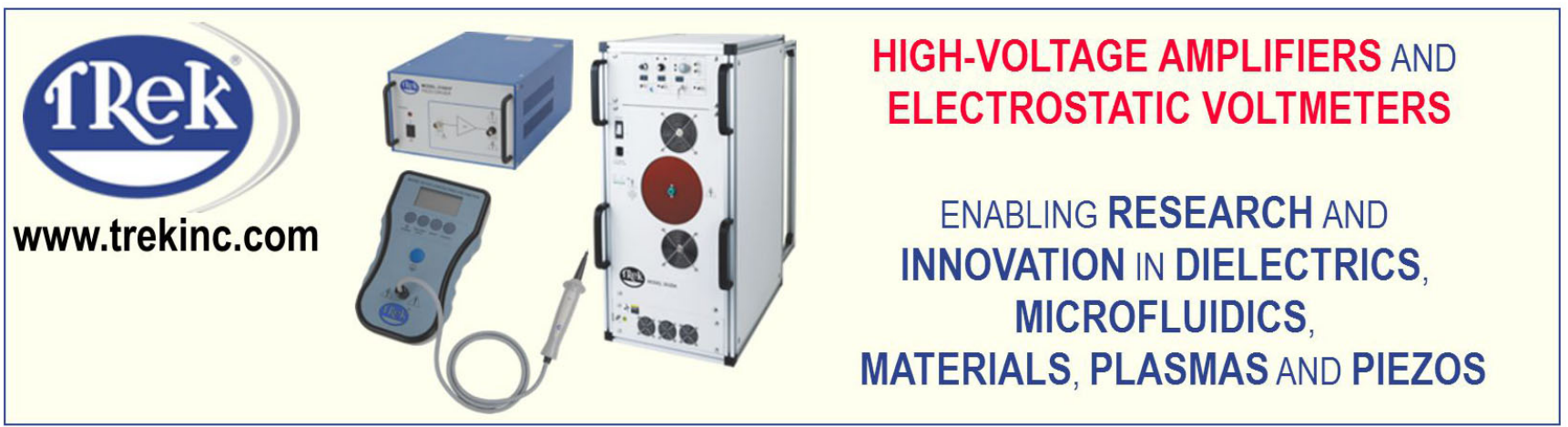




\title{
The ion velocity distribution function in a current-free double layer
}

\author{
Amy M. Keesee and Earl E. Scime \\ Department of Physics, West Virginia University, Morgantown West Virginia 26508 \\ Christine Charles, Albert Meige, and Rod Boswell \\ Space Plasma and Plasma Processing group, Plasma Research Laboratory, Research School of Physical \\ Sciences and Engineering, The Australian National University, Canberra ACT 0200, Australia
}

(Received 24 May 2005; accepted 20 July 2005; published online 26 August 2005)

\begin{abstract}
A portable, low-power, diode laser-based laser-induced fluorescence (LIF) diagnostic incorporating a heated iodine cell for absolute wavelength reference was installed on the Chi-Kung helicon source [K. K. Chi, T. E. Sheridan, and R. W. Boswell, Plasma Sources Sci. Technol. 8, 421 (1999)] to measure the ion velocity distribution function of argon ions as they transited a current-free double layer (DL) created where the solenoidal magnetic field diverges at the junction of the plasma source and the diffusion chamber. Based on LIF measurements of the transiting ion beam energy, the strength of the potential drop across the DL increases with decreasing neutral pressure and increasing magnetic field strength in the source. The location of the double layer also moves further downstream of the helicon source with increasing pressure. LIF measurements of the ion beam energy were found to be in good agreement with measurements obtained with a retarding field energy analyzer and also with numerical predictions. (C) 2005 American Institute of Physics.
\end{abstract} [DOI: $10.1063 / 1.2033647$ ]

\section{INTRODUCTION}

Recent experiments by a number of groups have demonstrated that electric double layers can form spontaneously in a current-free plasma expanding in a diverging magnetic field, i.e., a magnetic nozzle. ${ }^{1-5}$ Double layers (DLs) are narrow, local regions of strong electric potential gradient isolated from plasma boundaries. In the Charles and Boswell experiments, the combination of a strong gradient in the plasma potential and the observation of an ion beam in the diffusion region of a helicon plasma expanding in a divergent magnetic field demonstrated that a DL was created in the expanding plasma. ${ }^{1}$ Both the plasma potential and ion beam measurements were obtained with a retarding field energy analyzer (RFEA). ${ }^{6}$ In the Cohen et ll $^{4}{ }^{4}$ and Sun et al. ${ }^{5}$ expanding helicon plasma experiments, laser induced fluorescence (LIF) measurements of high speed ion flows, i.e., ion beams, were interpreted as evidence of the presence of DLs in the expanding plasmas.

Typical normalized RFEA ion energy distribution function (IEDF) measurements in the plasma downstream of the region of diverging magnetic field are shown in Fig. 1. The two ion populations evident in Fig. 1 (the two peaks in the IEDF) are the fast ion beam flowing away from the source and the stationary ion population trapped downstream by the strong plasma potential gradient of the DL. ${ }^{7}$ The trapped ion population appears in the grounded RFEA measurement at an energy equal to the local plasma potential while the ion beam appears at higher energy. However, the RFEA measurements are limited in spatial resolution (the RFEA can only be placed at discrete locations along the axis of the experiment) and there was a strong possibility that the grounded RFEA significantly perturbed the plasma during the measurement. In this work, we present spatially resolved, nonperturbative, LIF measurements of ion flow in the same experimental ap- paratus used for the RFEA measurements. The LIF diagnostic provides detailed measurements of ion acceleration through the DL and the corresponding presheath. The ion beam energies measured with LIF are consistent with the RFEA measurements as well as with the predictions of a numerical simulation-in both DL spatial structure and ion beam energy. LIF measurements were also performed with and without a RFEA-style probe in the plasma.

The portable LIF apparatus used for these experiments is the same diagnostic used in the Cohen et al. measurements of parallel ion flow speeds in the Magnetic Nozzle Experiment (MNX), a helicon discharge expanding through a magnetic nozzle. ${ }^{4}$ In those experiments, for a neutral pressure of $0.6 \mathrm{mTorr}$ in the main chamber, an ion beam of energy of 17 $\mathrm{eV}$ (corresponding to a beam speed of $9055 \mathrm{~m} / \mathrm{s}$ ) was observed. Of particular relevance to the measurements presented in this work was the observation of an exponential decrease in the LIF signal from the ion beam as a function of distance from the DL-even though the ion beam energy remained constant or increased slightly with distance from the DL. Analysis of the MNX measurements indicated that the decrease in LIF signal arose from the collisional depletion of the metastable ion states probed in the LIF measurement process, i.e., the beam continued to propagate into the diffusion region but the metastable ions in the beam needed for the LIF measurement were rapidly quenched.

In contrast to the MNX experiments, the Sun et al. experiments were performed in the freely expanding Hot hELicon eXperiment (HELIX) helicon plasma (whereas the expanding MNX plasma was channeled through a small aperture and a strong magnetic nozzle). With a geometry very similar to the Chi-Kung apparatus, high speed ion flows were again observed in HELIX below a critical neutral pressure. Subsequent LIF and Langmuir probe measurements in 

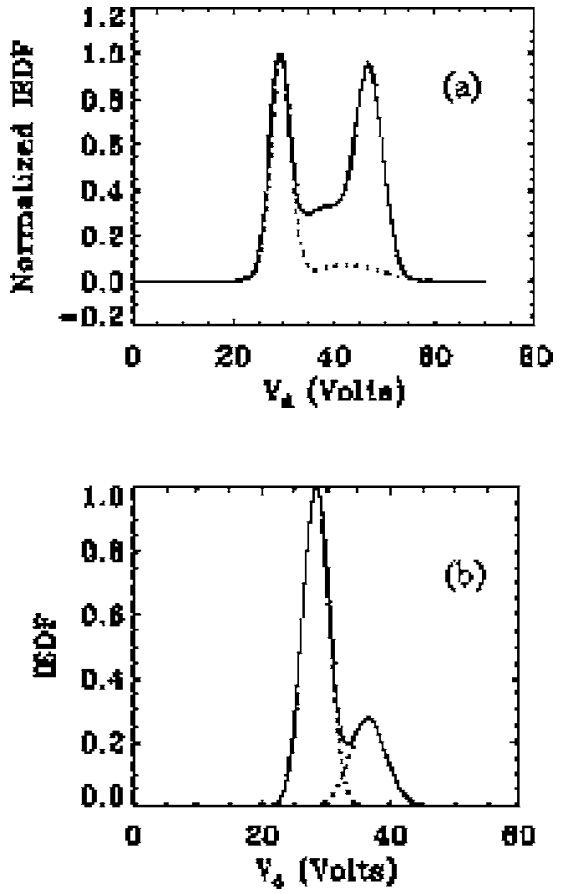

FIG. 1. Normalized IEDFs obtained with the RFEA at $P=250 \mathrm{~W}$ and the strong magnetic field case. (a) $z=37 \mathrm{~cm}$ (solid line) and $z=50 \mathrm{~cm}$ (dashed line) for $P=0.35$ mTorr. (Ref. 3). (b) $z=37 \mathrm{~cm}$ for $P=1 \mathrm{mTorr}$. The dashed line is a Gaussian fit to each peak.

HELIX provided detailed information about the spatial structure and magnitude of the electric field in the ion acceleration region. ${ }^{8}$ Consistent with the predictions of a onedimensional particle-in-cell (PIC) computer code that simulated an expanding, current-free plasma, ${ }^{9}$ the later HELIX measurements indicated that the ions accelerated through the presheath of the DL and, once in the DL proper, the ions rapidly accelerated to supersonic speeds. Downstream of the DL, a single Maxwellian, trapped population of ions was observed.

In this work, we report LIF measurements of the parallel ion velocity distribution function (IVDF) in the Chi-Kung helicon plasma source as a function of neutral pressure and magnetic field strength. The plasma source and LIF diagnostic are described in Sec. II. In Sec. III, the details of ion acceleration in the DL presheath and the DL are reviewed and compared to numerical models and RFEA probe measurements. Characteristics of the ion population trapped downstream of the DL by the potential gradient are also presented. Implications of the measurements and a relevant numerical model of an expanding plasma are discussed in Sec. IV.

\section{EXPERIMENTAL APPARATUS}

The Chi-Kung helicon source ${ }^{10}$ [Fig. 2(a)] consists of a $32 \mathrm{~cm}$ long, $15 \mathrm{~cm}$ diameter glass tube terminated at one end by a $1 \mathrm{~cm}$ thick glass plate. Attached to the tube opposite of the plate is a grounded aluminum diffusion chamber, $30 \mathrm{~cm}$ long with a $32 \mathrm{~cm}$ diameter. Rf power at $13.56 \mathrm{MHz}$ is supplied through a matching network to a $20 \mathrm{~cm}$ long double saddle antenna wrapped around the glass tube. Gas, typically

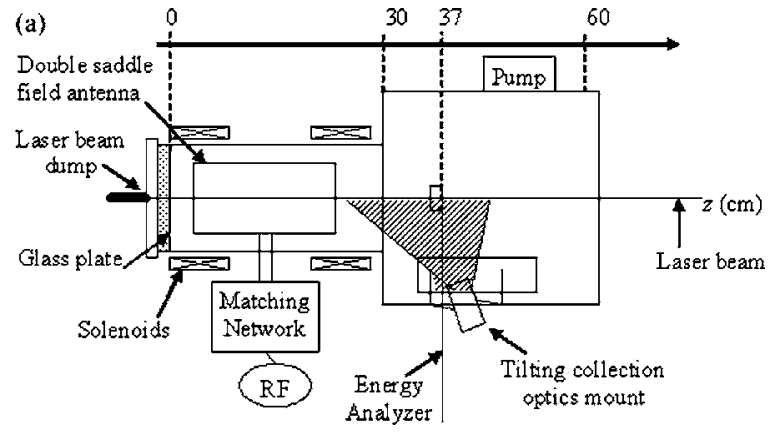

(b)

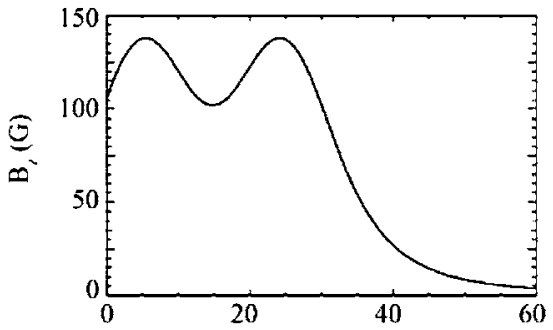

(c)

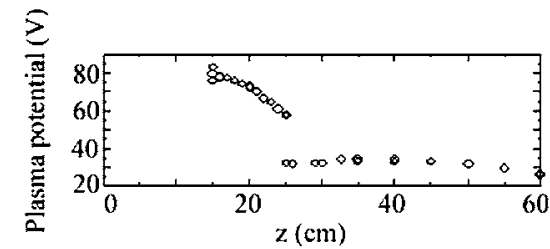

FIG. 2. (a) Chi-Kung apparatus with field of view of LIF collection optics shown as the shaded region. (b) Magnetic field geometry shown for strong magnetic field $\left(I_{B}=6 \mathrm{~A}\right.$ in both coils) case. (c) Plasma potential versus axial position in Chi-Kung showing double layer potential drop for $P$ $=0.2 \mathrm{mTorr}, P=250 \mathrm{~W}$, and strong magnetic field case (Ref. 1).

argon, is introduced through the side of the diffusion chamber. A base pressure of $2 \times 10^{-6}$ mTorr is obtained by a turbomolecular/rotary pumping system connected to the wall of the diffusion chamber. The magnetic field is created by two co-axial, solenoidal coils placed at each end of the source tube. Varying magnetic field strengths and profiles were created by independently adjusting the current to each coil. The highest field case investigated (peak field of $140 \mathrm{G}$ ) is obtained for a current of 6 Amps in both coils. There are no coils around the diffusion chamber, yielding a field geometry as shown in Fig. 2(b) for the strong magnetic field case. Neutral pressure in the diffusion chamber is measured with an ion gauge and a Baratron ${ }^{\circledR}$ gauge.

For neutral pressures less than about 1.0 mTorr, a strong gradient in the plasma potential spontaneously appears near the end of the helicon plasma source. The plasma potential as a function of axial position is shown in Fig. 2(c) for a pressure of 0.2 mTorr. The RFEA probe provides measurements of the local plasma potential and the ion energy distribution function (IEDF). Two such IEDF measurements are shown in Fig. 1. The IEDF measured at $z=37 \mathrm{~cm}$ (solid line) and $z$ $=50 \mathrm{~cm}$ (dashed line) for a pressure of 0.35 mTorr are shown in Fig. 1(a). At $z=37 \mathrm{~cm}$, the ion beam is approximately $9300 \mathrm{~m} / \mathrm{s}$ faster than the trapped ion population. As the neutral pressure increases, both the energy and density of the ion beam decrease. The normalized IEDF for a pressure of 1.0 mTorr at $z=37 \mathrm{~cm}$ is shown in Fig. 1(b). At this higher pressure, the beam is approximately $6200 \mathrm{~m} / \mathrm{s}$ faster than the 
background population. According to previous RFEA measurements, collisions reduce the ion beam below the detection threshold by $z=50 \mathrm{~cm}$, as a result of ion-neutral collisions. $^{3}$

LIF measurements of argon ions were performed with a portable, tunable-diode-laser system. ${ }^{11}$ The laser, tuned to $668.6138 \mathrm{~nm}$, pumps argon ions in the $3 \mathrm{~d}^{4} \mathrm{~F}_{7 / 2}$ metastable state to the $4 \mathrm{p}^{4} \mathrm{D}_{5 / 2}$ state. These ions can then decay to the $4 \mathrm{~s}^{4} \mathrm{P}_{3 / 2}$ state, emitting fluorescence radiation at $442.7244 \mathrm{~nm}$. This radiation is collected by a focused set of optics and directed into a bandpass filtered photomultiplier tube (PMT) via fiber optic cable. The PMT signal is measured with a lock-in amplifier referenced to a mechanical chopper that modulates the laser beam before it enters the plasma. For each measurement the laser wavelength is swept through a $0.015 \mathrm{~nm}(10 \mathrm{GHz})$ window centered on the Doppler-shifted (due to bulk ion flow) absorption wavelength. On the ChiKung source, the laser enters through a window in the center of the flange at the end of the diffusion chamber. An alternate gas inlet on the end of the source is used as a beam dump to minimize reflections. The fluorescence radiation is collected by a set of focused optics mounted to a rectangular window on the side of the diffusion chamber. The optics mount can be tilted to select the axial position, corresponding to $z$ $=25-45 \mathrm{~cm}$ along the axis. Based on the core size of fiber optic cable used and magnification of the optics, spatial resolution is approximately $1 \mathrm{~cm}$ along the laser beam.

The total absorption line shape for typical helicon source parameters consists of 18 Zeeman split components that are Doppler broadened. For this LIF scheme, the fluorescence line has six $\pi$ transitions $(\Delta M=0 ; M$ is the magnetic orbital quantum number) and $12 \sigma$ transitions (six with $\Delta M=+1$, six with $\Delta M=-1$ ). For parallel (to the magnetic field) injection used in these experiments, only the $\sigma$ transitions are pumped. The Zeeman broadening of the $\sigma$ lines due to a $1 \mathrm{k}$ Gauss magnetic field is approximately $9.0 \times 10^{-4} \mathrm{~nm}$ $(\sim 0.6 \mathrm{GHz}){ }^{12}{ }^{12}$ For the magnetic fields used in this experiment $(\leqslant 150 \mathrm{G})$ Zeeman broadening can be ignored. Thus, the intensity line shape can be described by a single Dopplershifted and broadened peak:

$$
I_{R}(\nu)=I_{R}\left(\nu_{0}\right) \exp \left[\frac{-\left(\nu-\nu_{0}-V_{0} \nu_{0} / c\right)^{2}}{\alpha_{D} T_{i}}\right],
$$

where $I_{R}(\nu)$ is the measured LIF signal, $\left(V_{0} \nu_{0} / c\right)$ is the overall Doppler shift of the distribution due to bulk flow of the ions, $\alpha_{D}$ scales the width of the thermal broadening for argon ions of mass $m_{i}\left(\alpha_{D}=2 k \nu_{0}^{2} / m_{i} c^{2}\right)$, and $T_{i}$ is the temperature of the ions in eV. Fits of Eq. (1) to the measured LIF intensity as a function of laser frequency yield ion temperatures and ion distribution shifts with average precisions of $\pm 0.0043 \mathrm{eV}$ and $\pm 0.27 \mathrm{GHz}$, respectively. Additional details of the argon ion LIF measurement technique can be found in Refs. 4, 11, and 13.

To provide an absolute zero velocity reference for every LIF measurement, the laser was directed through a heated iodine cell. ${ }^{14}$ The fluorescence spectrum of the iodine cell is recorded with an amplified photodiode for each LIF laser scan and used to calculate the shift, $\Delta \nu$, of the central LIF

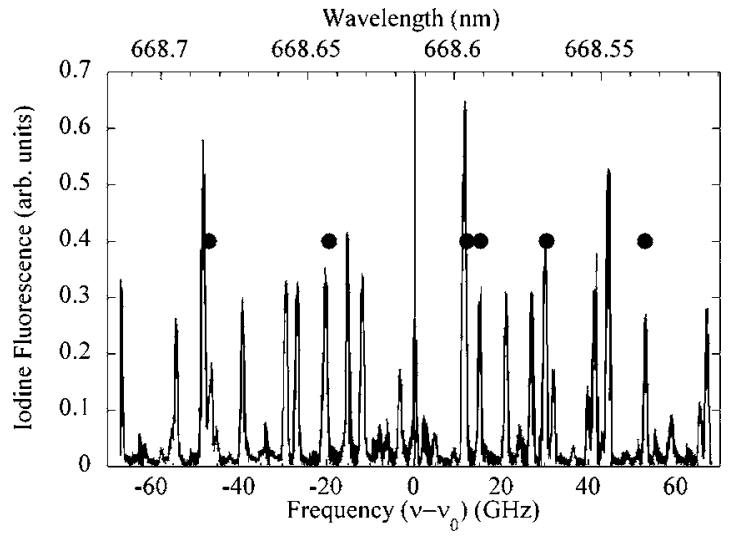

FIG. 3. Iodine fluorescence spectrum for $60 \mathrm{GHz}$ on either side of the Ar II pump line. The vertical line at zero GHz indicates the Ar II pump line. The solid dots indicate iodine absorption lines in the published line atlas (Ref. 15).

peak from $\lambda_{0}=668.6138 \mathrm{~nm}$. A shift of the center of the LIF peak to lower frequency indicates a bulk flow of the ions toward the laser (away from the source). The parallel ion flow speed is given by $\nu_{0}=\lambda_{0} \Delta \nu$. A $120 \mathrm{GHz}$ wide reference iodine fluorescence spectrum ${ }^{14}$ is shown in Fig. 3. The Ar II pump line is indicated by a vertical line at zero GHz. As in Fig. 3 of Ref. 13, the locations of molecular iodine absorption lines in the published atlas ${ }^{15}$ are indicated by solid circles. Note that most of the molecular iodine fluorescence lines do not appear in the standard atlas of absorption lines. This discrepancy is not restricted to only weak lines as a number of the fluorescence lines that are missing from the absorption atlas are "stronger" than those that do correspond to absorption lines in the atlas. A possible explanation for this could be that additional vibrational modes are excited by heating the iodine cell.

For most measurements reported here, the molecular iodine fluorescence peak at $668.6196 \mathrm{~nm}(3.89 \mathrm{GHz}$ from the rest frame Ar II absorption line) was used as a zero-velocity reference for the LIF measurement. For plasma conditions that yielded higher flow speeds, the molecular iodine fluorescence peak at $668.6318 \mathrm{~nm}(12.1 \mathrm{GHz}$ from the rest frame Ar II absorption line) was used. The absolute wavelengths of the iodine cell peaks were measured with an accuracy of $\pm 0.0004 \mathrm{~nm}$, yielding an error in the absolute bulk ion flow speed determination of $\pm 180 \mathrm{~m} / \mathrm{s}$. This systematic error is combined with the statistical error in determining the center of LIF peak (approximately $\pm 20 \mathrm{~m} / \mathrm{s}$ on average) for the total parallel ion flow speed error shown in Figs. 8 and 9 (typically $\pm 181 \mathrm{~m} / \mathrm{s}$ ).

As an aside we note that coarse tuning of the diode laser was accomplished with both a Burleigh WA-4500 and a Burleigh WA-1100 wavemeter. The first unit is intended for use for a pulsed laser and the second with lasers in the wavelength range $700-1650 \mathrm{~nm}$. Although both provided wavelength measurements of the $668 \mathrm{~nm}$ tunable diode laser, we found that the units disagreed by at least $\sim 0.05 \mathrm{~nm}$ $(\sim 33.5 \mathrm{GHz})$. Therefore, fine tuning of the diode laser was accomplished entirely by comparing the observed molecular iodine fluorescence spectrum to previous measurements. ${ }^{14}$ 


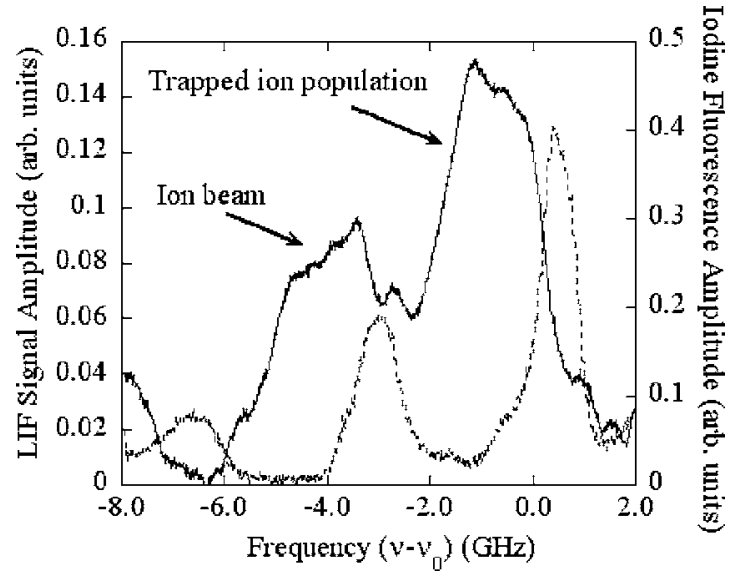

FIG. 4. LIF signal versus laser frequency showing the beam and trapped ion populations (solid line). Also shown is the iodine cell fluorescence spectrum for the same scan of the laser frequency (dashed line). ( $0 \mathrm{GHz}$ on the abscissa corresponds to $\nu_{0}$, the zero-velocity location of the line center.)

Because low plasma densities $\left(\sim 10^{9} \mathrm{~cm}^{-3}\right.$ in the diffusion chamber) required long lock-in integration times $(\sim 1 \mathrm{~s})$ to differentiate background plasma emission from the laser-induced fluorescence, at least five one-minute laser frequency scans were needed to obtain adequate signal-tonoise. Rf powers greater than $400 \mathrm{~W}$ were required to obtain LIF signal and rf powers $>750 \mathrm{~W}$ were problematic given the need to operate the source long enough to acquire a complete LIF scan (at rf powers greater than $750 \mathrm{~W}$ the matching circuit and rf antenna would overheat during the measurement). Therefore, most measurements reported here were performed at a rf power of $740 \mathrm{~W}$. Except for the magnetic field strength parameter scan, the source was operated in either the strong magnetic field case with $I_{B}=6 \mathrm{Amps}$ in each coil [Fig. 1(b)] or in the weak magnetic field case with $I_{B}$ $=3$ Amps in each coil. For all source conditions, the best signal-to-noise was obtained at $z=25 \mathrm{~cm}$, just inside the source.

\section{ION VELOCITY DISTRIBUTION MEASUREMENTS}

An ion velocity distribution measured with LIF at an axial position of $z=31 \mathrm{~cm}$ for a pressure of $1.3 \mathrm{mTorr}$ and for the low magnetic field strength configuration is shown in Fig. 4. Two distinct peaks are observed: the trapped ion population near the unshifted line center and the ion beam shifted to lower frequency (higher velocity). Also shown is the simultaneous iodine cell fluorescence measurement used to determine the line center.

The log of LIF signal versus parallel ion flow speed and axial position for the same plasma parameters as Fig. 4 is shown in Fig. 5(a). From $z=25 \mathrm{~cm}$ to $30 \mathrm{~cm}$, the LIF measurements show an ion population accelerating from approximately $2000 \mathrm{~m} / \mathrm{s}$ to $4000 \mathrm{~m} / \mathrm{s}$. Defining the DL location as that point in the plasma where the trapped ion population appears (as seen in Fig. 4 which is a slice through the data of Fig. 5 at $z=31 \mathrm{~cm}$ ), the LIF measurements yield a DL location of approximately $z=28 \mathrm{~cm}$ for the low magnetic field strength case. The $z=25 \mathrm{~cm}$ DL location measured by Charles and Boswell [Fig. 1] was obtained for the strong

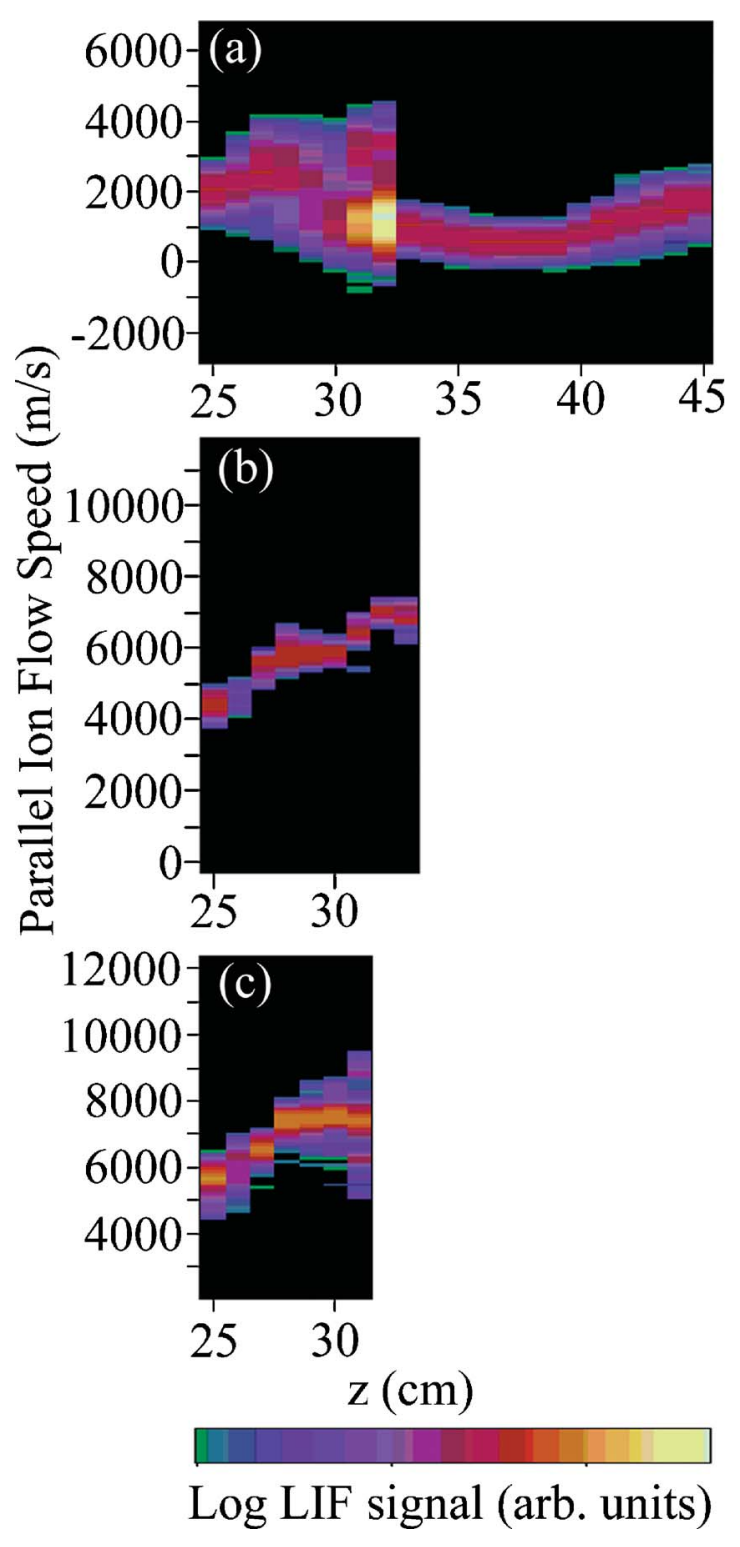

FIG. 5. (Color) Logarithm of LIF signal versus parallel argon ion flow speed (away from the source) and axial position for (a) $P=1.3$ mTorr, weak magnetic field case, (b) $P=0.55$ mTorr, strong magnetic field case, and (c) $P=0.37$ mTorr, strong magnetic field case. rf power is $740 \mathrm{~W}$ in all cases.

magnetic field strength case and at significantly lower neutral pressure $(0.2$ mTorr $)$. At similar neutral pressures ( $\sim 1.0 \mathrm{mTorr})$, the RFEA probe indicates an ion beam energy of $8 \mathrm{eV}$ (corresponding to $\sim 6200 \mathrm{~m} / \mathrm{s}$ ) and a beam density of a few $10^{9} \mathrm{~cm}^{-3}$. Since there was a chance that the RFEA probe, with its grounded and conducting shaft, could perturb the plasma, LIF measurements were taken with and without a mock RFEA inserted in the plasma. The LIF measurements were unaffected by the presence of the probe located $12.6 \mathrm{~cm}$ downstream of the LIF interrogation volume.

For lower neutral pressures $(<1 \mathrm{mTorr})$ and for the strong magnetic field case, useful LIF signal was only achieved between axial positions $z=25-32 \mathrm{~cm}$. Below neutral pressures of 0.35 mTorr, LIF measurements were not possible anywhere along the axis of the experiment-most likely due to significantly reduced plasma densities downstream of the DL at low neutral pressure. 


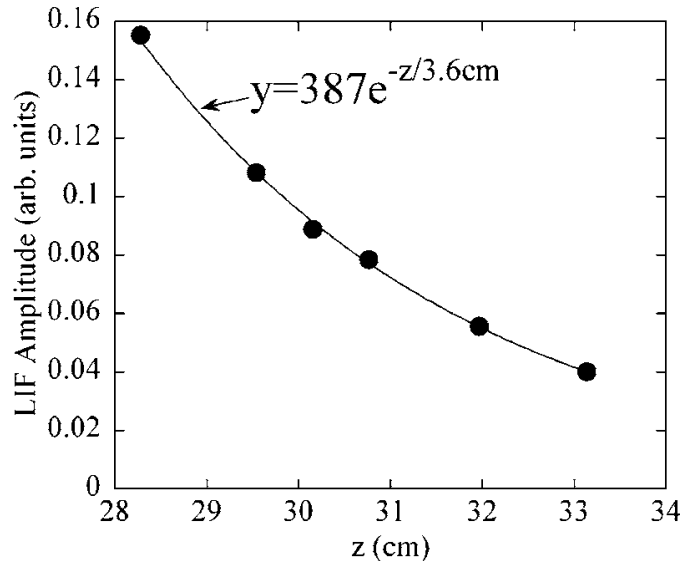

FIG. 6. LIF signal amplitude of ion beam and exponential fit versus axial position from data of Fig. 5(a).

Figures 5(b) and 5(c) show the log of LIF signal versus parallel ion flow speed and axial position for neutral pressures of 0.55 mTorr and 0.37 mTorr, respectively. Note that the ion flow speed upstream of the DL increases as the pressure decreases, indicating a larger potential difference across the DL for lower pressures. For $P=0.55 \mathrm{mTorr}$ and $P$ $=0.37$ mTorr, the maximum ion speeds measured by LIF were approximately $7000 \mathrm{~m} / \mathrm{s}$ and $7300 \mathrm{~m} / \mathrm{s}$ (corresponding to ion beam energies of $10.1 \mathrm{eV}$ and $11.0 \mathrm{eV}$ ), respectively. Based on the measured downstream electron temperatures of $7 \mathrm{eV}$ and $8 \mathrm{eV}$, respectively, the ion flow speeds are clearly supersonic compared to ion sound speeds of $5300 \mathrm{~m} / \mathrm{s}$ for $0.55 \mathrm{~m}$ Torr and $5700 \mathrm{~m} / \mathrm{s}$ for 0.33 mTorr. Since the LIF signal was lost near the DL, the final ion flow speeds could not be measured with LIF. We expect that additional ion acceleration up to similar energies as measured by the RFEA occurred in the DL-which would be consistent with the RFEA probe measured ion beam energies downstream of the DL at $z=37 \mathrm{~cm}: 13.4 \mathrm{eV}$ at 0.55 mTorr (obtained at a rf power of $250 \mathrm{~W}$ and not the $740 \mathrm{~W}$ used here) and $15.2 \mathrm{eV}$ at 0.33 mTorr. These spatially resolved LIF measurements of the IVDF also show that as the neutral pressure increases, the DL forms further inside the diffusion chamber (based on the ion beam acceleration continuing further downstream for higher pressures).

The LIF intensity of the ion beam population measured in Fig. 5(a) decreases rapidly with distance downstream of the DL. The amplitude of the LIF signal for the ion beam population is shown as a function of distance downstream of the DL in Fig. 6. An exponential fit to the measurements yields a $1 / e$ folding distance of $3.6 \mathrm{~cm}$. Assuming that the decrease is due to a rapidly decreasing population of metastable ions in the beam and the rapidly expanding magnetic field (decreasing plasma density), the $1 / e$ folding distance provides a measure of the quenching rate of the metastable ions. The $1 / e$ folding distance for the ion beam signal obtained in these measurements is inconsistent with what was observed in MNX (1.6 cm for a pressure of $0.135 \mathrm{mTorr})^{4}$ and other argon LIF experiments. ${ }^{16}$ Using the $1 / e$ folding distance theoretically predicted by Cohen et al. ${ }^{4}$ one would expect to find a $1 / e$ folding distance of $0.4 \mathrm{~cm}$ for a pressure

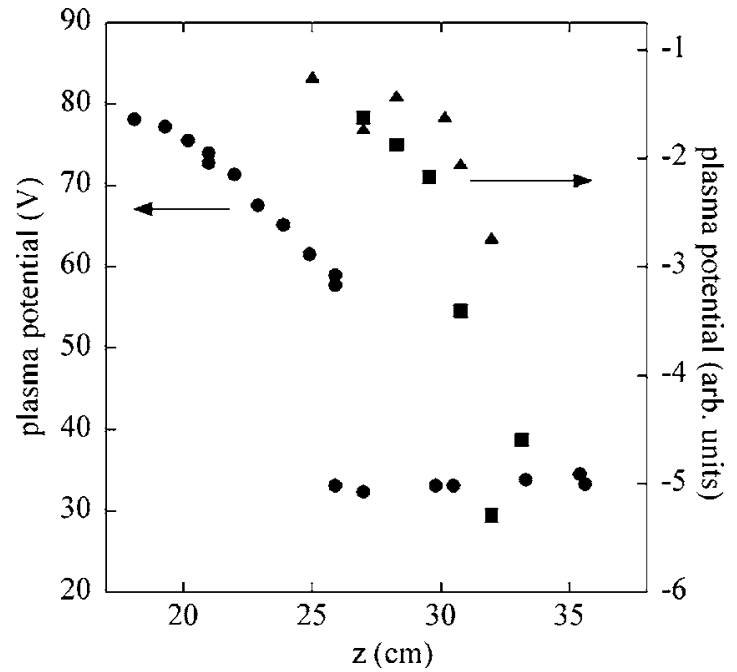

FIG. 7. Plasma potential estimated from ion beam energy versus position from data of Fig. 5(a) (triangles) and Fig. 5(b) (squares). Plasma potential versus position measured by RFEA at 0.2 mTorr (circles) (Ref. 1).

of 1.3 mTorr in Chi-Kung, assuming the same collisional quenching cross section for the metastable state. In other words, the LIF signal persists much further downstream of the DL in Chi-Kung than expected. The very low pressure MNX experimental configuration included a physical aperture that limited plasma flow and therefore plasma production downstream of the DL. The Chi-Kung experiment has no aperture and we hypothesize that the longer $1 / e$ folding distance results from new metastable production by high energy electrons throughout the measurement region in this higher pressure plasma. As noted above, at lower neutral pressures, the LIF signal decreases much more rapidly downstream of the DL.

Assuming energy conservation, changes in the measured ion beam energy can provide a measurement of the local gradient in the plasma potential. The spatial profile of the plasma potential, as estimated from the ion beam energy, is shown in Fig. 7 for the LIF data of Figs. 5(a) and 5(b). Also shown, for comparison, is the plasma potential measured by RFEA at 0.2 mTorr [Fig. 2(c)]. It can be seen that the plasma potential in the presheath of the DL has a steeper gradient at lower pressures. Also, the location of the DL appears to move further downstream of the source as the pressure increases.

A pressure scan at $z=25 \mathrm{~cm}$ (just upstream of the DL), $\mathrm{rf}$ power $=740 \mathrm{~W}$, and for the strong magnetic field case is shown in Fig. 8. As in Fig. 5, there is a substantial increase in the parallel ion flow speed into the double layer with decreasing pressure. A similar scaling of ion flow speed on inverse neutral pressure was reported by Sun et al. in a higher density, expanding helicon source plasma. ${ }^{5}$ At the lowest neutral pressure, the ion flow speed reaches $6000 \mathrm{~m} / \mathrm{s}$ well upstream of the DL. As can be seen in Fig. 5(c), at the lowest neutral pressure of 0.37 mTorr the parallel ion speed reaches nearly $8000 \mathrm{~m} / \mathrm{s}$ at the furthest downstream location with useful LIF emission. That the ion beam energy scales as $1 / P_{0}^{2}$ with the neutral pressure, suggests that the double layer strength also scales with $1 / P_{0}^{2}$. 


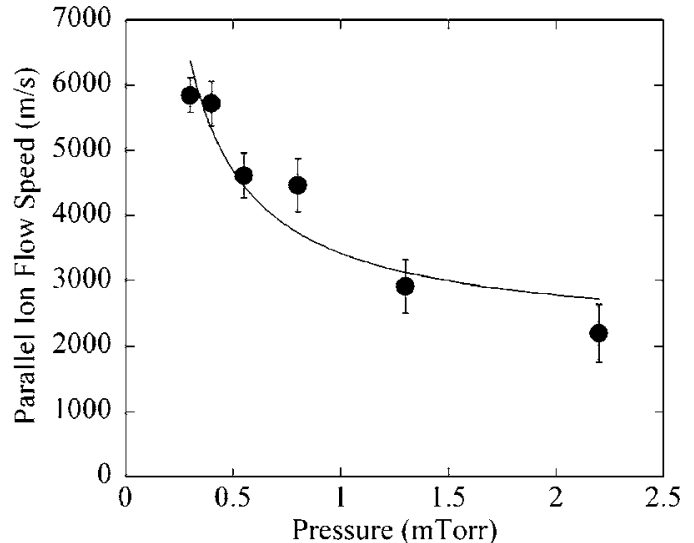

FIG. 8. Parallel ion flow speed versus neutral pressure at $z=25 \mathrm{~cm}$, rf power $=740 \mathrm{~W}$, and for the strong magnetic field case. The solid line is given by $v_{i 0}=\left(1267 / P_{0}+2156\right) \mathrm{m} / \mathrm{s}$.

In Fig. 9(a), the parallel ion flow speed is shown as a function of magnetic field strength for the same current in both coils. In Fig. 9(b), the ion flow speed measurements are for the case where the top coil (furthest from the diffusion chamber) was held at 6 Amps while the current through the bottom coil was varied. Note that a current of 6 Amps in both coils yields the strong magnetic field case geometry shown in Fig. 2(b). Because Fig. 9(a) indicates that as the magnetic field strength increases the ions flow into the diffusion cham-
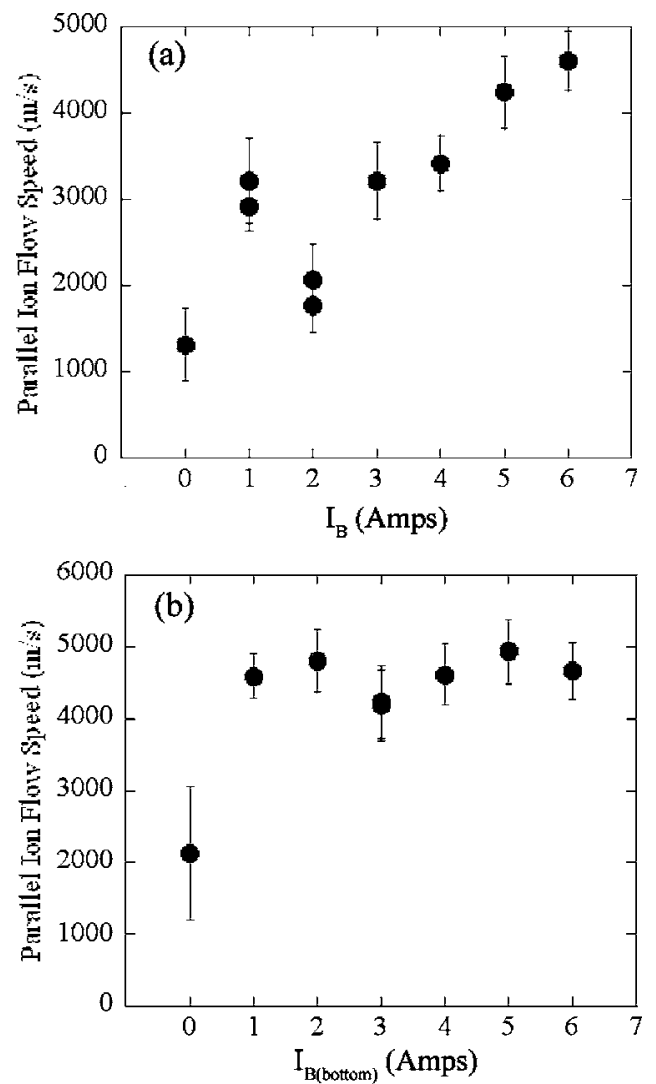

FIG. 9. Parallel ion flow speed versus magnet coil current (magnetic field) for (a) equal currents in both coils and (b) top coil held at 6 Amps while current through bottom coil was varied. rf power of $740 \mathrm{~W}, P$ $=0.55 \mathrm{mTorr}$, and $z=25 \mathrm{~cm}$.

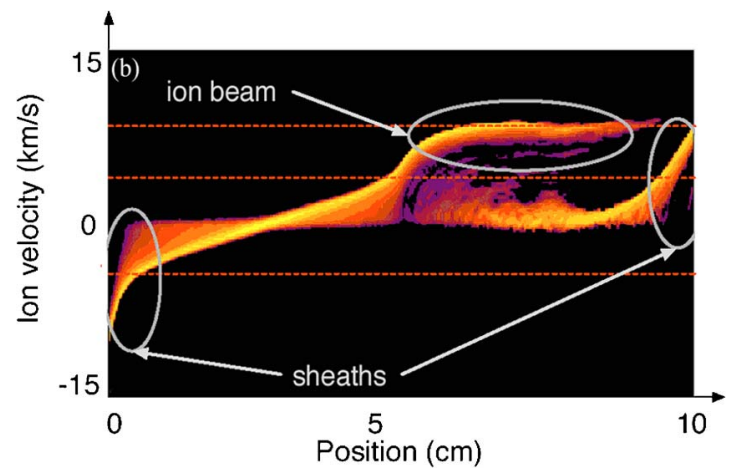

FIG. 10. (Color online) Parallel ion velocity distribution function along MCC-PIC simulation axis (Ref. 20).

ber faster, it would be natural to assume that the increased ion flow arises from magnetic moment conservation leading to increased conversion of perpendicular thermal energy into directed parallel ion flow. However, the measurements shown in Fig. 9(b) demonstrate that it is the coil furthest from the diffusion chamber that has the most influence over the ion flow speed into the diffusion chamber. Thus, the strength of the double layer is controlled by the plasma source parameters (i.e., the magnetic field strength in the source), and the high speed ion flows result from acceleration in the spontaneously formed DL and not any magnetic moment conservation process in the diverging magnetic field. The dependence of the potential drop across the DL on the coil furthest from the diffusion chamber is confirmed by RFEA measurements. ${ }^{17}$

\section{DISCUSSION}

The high spatial and velocity resolution of the LIF IVDF measurements shown in Fig. 5 provide a unique opportunity for comparison with numerical predictions of the structure and magnitude of DL formation in expanding, current-free plasmas. A recently developed one-dimensional Monte Carlo Collision ${ }^{18}$ particle-in-cell $^{19}$ (MCC-PIC) plasma computer code was used to confirm that DLs could form in current-free expanding plasmas. ${ }^{20}$ The PIC simulation consisted of a bounded plasma with a floating left wall and a grounded right wall. The system was separated into two regions: the source region and the diffusion chamber. In the source region, the electrons are heated up by a uniform rf electric field of $10 \mathrm{MHz}$ perpendicular to the axis of the simulation. In the diffusion chamber, the diffusion of the plasma in the diverging magnetic field was modeled with a simple, spatially dependent, loss mechanism.

The ion velocity distribution in phase space, where the abscissa represents the position and the ordinate the ion velocity, predicted by the PIC code is shown in Fig. 10 for a simulation neutral pressure of 1 mTorr. Throughout the simulation, a low energy population of ions is observed which corresponds to the ions which are created by ionization and charge exchange collisions. Downstream of the DL a high energy population can be seen which corresponds to the ions accelerated while traversing the potential drop of the DL. Note that the acceleration of the ions occurs over many cen- 
timeters in the simulation (in the presheath and the sheath) while the actual DL is much narrower and appears in the ion phase space plot as a narrow region of strong ion acceleration. The acceleration of the background ion population to the boundaries of the simulation volume (as the ions fall through the sheath) is also evident at the left and right sides of Fig. 10. The spatial structure, beam energy, character of ion acceleration region, and ion heating in the presheath in the simulation are all consistent with the LIF measurements shown in Fig. 5(a).

In this work we have reported measurements of the parallel ion flow speed in the Chi-Kung helicon plasma source using laser-induced fluorescence. The LIF measurements show acceleration of ions into the double layer, as well as a fast ion beam and slow trapped ion population downstream of the double layer. We have found that the potential drop of the DL increases with decreasing pressure and the LIF ion beam energy measurements agree with previous RFEA measurements. The LIF measurements also demonstrated that the presence of the RFEA probe did not significantly affect the magnitude of the observed ion beam energy. Perhaps most significantly, the dependence of the ion beam energy on the upstream plasma conditions and not the details of the magnetic field structure in the expansion region suggest that optimal ion acceleration can be achieved through optimization of the upstream plasma parameters. In future experiments we intend to investigate the off-axis parallel ion flow to better understand the radial distribution of the ions and to perform argon neutral LIF measurements to investigate the possibility of momentum coupling to the neutrals (plasma detachment) through charge-exchange collisions with ions.

\section{ACKNOWLEDGMENTS}

We thank P. Alexander for technical assistance and the Laser Physics and Atomic and Molecular Physics Depart- ments of the RSPhysSE for the loan of critical equipment at ANU.

This work was supported by NSF Grant PHY-0315356, and the NSF EAPSI program in cooperation with Australian Academy of Science. A.M.K. was also supported by the DOE Fusion Energy Science Fellowship program.

${ }^{1}$ C. Charles and R. W. Boswell, Appl. Phys. Lett. 82, 1356 (2003).

${ }^{2}$ C. Charles, Appl. Phys. Lett. 84, 332 (2004).

${ }^{3}$ C. Charles and R. W. Boswell, Phys. Plasmas 11, 3808 (2004).

${ }^{4}$ S. A. Cohen, N. S. Siefert, S. Stange, E. E. Scime, R. F. Boivin, and F. Levinton, Phys. Plasmas 10, 2593 (2003).

${ }^{5}$ X. Sun, C. Biloiu, R. Hardin, and E. Scime, Plasma Sources Sci. Technol. 13, 359 (2004).

${ }^{6}$ C. Charles, A. W. Degeling, T. E. Sheridan, J. H. Harris, M. A. Lieberman, and R. W. Boswell, Phys. Plasmas 7, 5232 (2000).

${ }^{7}$ C. Charles and R. W. Boswell, Phys. Plasmas 11, 1706 (2004).

${ }^{8}$ X. Sun, A. Keesee, C. Biloiu, E. Scime, A. Meige, C. Charles, and R. Boswell, "Observations of ion-beam formation in a current-free doublelayer," Phys. Rev. Lett. 95, 025004 (2005).

${ }^{9}$ A. Meige, R. Boswell, C. Charles, J.-P. Boeuf, G. Hagelaar, and M. Turner. IEEE Trans. Plasma Sci. 33, 334 (2005).

${ }^{10}$ K. K. Chi, T. E. Sheridan, and R. W. Boswell, Plasma Sources Sci. Technol. 8, 421 (1999).

${ }^{11}$ R. F. Boivin and E. E. Scime, Rev. Sci. Instrum. 74, 4352 (2003).

${ }^{12}$ See EPAPS Document No. E-PHPAEN-10-003306, for "Zeeman splitting for LIF transitions and de-convolution technique to extract ion temperature." This document can be reached via a direct link in the online article's HTML reference section or via the EPAPS homepage (http://www.aip.org/ pubservs/epaps.html).

${ }^{13}$ G. D. Severn, D. A. Edrich, and R. McWilliams, Rev. Sci. Instrum. 69, 10 (1998).

${ }^{14}$ A. M. Keesee, E. E. Scime, and R. F. Boivin, Rev. Sci. Instrum. 75, 4091(2004).

${ }^{15}$ S. Gerstenkorn and P. Luc, Atlas Du Spectre D'absorption De La Molecule D'iode (Editions DuCentre National De La Recherche Scientifique, 1978).

${ }^{16}$ F. Skiff, G. Bachet, and F. Doveil, Phys. Plasmas 8, 3139 (2001).

${ }^{17}$ C. Charles, Phys. Plasmas 12, 044508 (2005).

${ }^{18}$ V. Vahedi and M. Surendra, Comput. Phys. Commun. 87, 179 (1995).

${ }^{19}$ C. K. Birdsall and D. Fuss, J. Comput. Phys. 3, 494 (1969).

${ }^{20}$ A. Meige, R. W. Boswell, C. Charles, and M. Turner, Phys. Plasmas 12, 052317 (2005). 